\title{
Association between macrophage migration inhibitory factor in the endometrium and estrogen in endometriosis
}

\author{
XIAO ZHANG ${ }^{1}$ and LIN MU $^{2}$ \\ ${ }^{1}$ Department of Gynecology, Sir Run Run Shaw Hospital, School of Medicine; ${ }^{2}$ Department of Gynecology, \\ The Second Affiliated Hospital, School of Medicine, Zhejiang University, \\ Hangzhou, Zhejiang 310000, P.R. China
}

Received July 24, 2014; Accepted April 1, 2015

DOI: $10.3892 /$ etm.2015.2516

\begin{abstract}
Recent studies have shown that macrophage migration inhibitory factor (MIF) has a possible role in endometriosis-related pain and infertility, yet it has not been explored whether the mRNA level of MIF is altered in endometrial tissues from patients with endometriosis. The aim of the present study was to compare the expression of MIF in endometrial tissues from women with and without endometriosis, and to analyze the association between endometrial MIF expression and $17 \beta$-estradiol $\left(\mathrm{E}_{2}\right)$. The protein and mRNA expression of MIF in the human endometrial tissue was assessed by western blotting and reverse transcription-polymerase chain reaction analysis, respectively. The MIF expression of women with endometriosis was found to be significantly higher than that of the controls. A positive correlation was noted between the serum $\mathrm{E}_{2}$ level and MIF expression. In endometrial cells from women with endometriosis, the level of $\mathrm{E}_{2}$-induced MIF upregulation was significantly higher than that in cells from women without endometriosis. In conclusion, this study demonstrated a significant increase in MIF expression in the endometrial tissues of women with endometriosis and an association between MIF expression and $\mathrm{E}_{2}$ level. MIF expression in endometrial cells from patients with endometriosis showed an increased sensitivity to stimulation by $\mathrm{E}_{2}$.
\end{abstract}

\section{Introduction}

Endometriosis, a condition characterized by the presence and proliferation of endometrial tissue outside the uterine cavity, is

Correspondence to: Professor Lin Mu, Department of Gynecology, The Second Affiliated Hospital, School of Medicine, Zhejiang University, 88 Jiefang road, Hangzhou, Zhejiang 310000, P.R. China E-mail: mulinzi5322@126.com

Abbreviations: $\mathrm{E}_{2}$, $17 \beta$-estradiol; MIF, macrophage migration inhibitory factor

Key words: endometriosis, eutopic endometrium, macrophage migration inhibitory factor, estrogen, $17 \beta$-estradiol a cause of pain and reduced fertility worldwide. The etiology of the condition is believed to involve the retrograde flow of menstrual fluid through the fallopian tubes, leading to the deposition of viable endometrial tissue and the subsequent implantation of this tissue onto the peritoneal surface (1). Although retrograde menstruation appears in $90 \%$ of women of a reproductive age, only $10 \%$ of cases are characterized by the presence of endometrial tissue outside the uterine cavity (2). It has previously been found that women with endometriosis exhibit aberrant endometrial gene expression (3). Furthermore, the endometrial cells from women with endometriosis have a predisposition to adhere and grow outside of the uterus (4).

Macrophage migration inhibitory factor (MIF) is a cytokine that is secreted by the immune cells and the anterior pituitary gland (5). MIF has been found to be a key mediator of systemic inflammatory responses and is thus a critical regulator of inflammatory pathways (6), which have an essential role in the pathogenesis of endometriosis (7). Compared with the MIF expression in normal fertile women, MIF expression has been found to be elevated in early, active endometriotic lesions and in the intrauterine human endometrium of women with endometriosis (8), which suggests that MIF has a possible role in endometriosis-related pain and infertility. Although the mechanism underlying the regulation of MIF is important, it has yet to be elucidated whether the mRNA level of MIF is altered in endometrial tissues from women with endometriosis. Endometriosis can be considered to be an estrogen-dependent disease (9) that is rarely observed prior to menarche and typically disappears following menopause. The aims of the present study, therefore, were as follows: i) To examine whether the MIF mRNA level is altered in endometrial tissues from women with endometriosis; ii) to investigate whether the MIF expression level in endometrial tissues is associated with the serum $17 \beta$-estradiol $\left(\mathrm{E}_{2}\right)$ level; and iii) to observe whether $\mathrm{E}_{2}$ treatment induces a change in MIF expression in endometrial cells.

\section{Materials and methods}

Patients and sample collection. This study was approved by the Ethics Committee of the School of Medicine, Zhejiang University (Hangzhou, China). Written informed consent was obtained from each subject prior to tissue collection. A total 
of 102 women of a reproductive age volunteered for this study. All subjects had a normal menstrual cycle (28-32 days) and had not received any anti-inflammatory or hormonal treatment for $\geq 6$ months before inclusion in the study. At the time of surgery, the pelvic organs of the women were examined carefully for the presence and extent of endometriosis.

Among the patients, 55 women (aged 25-39 years) were diagnosed with an ovarian endometriotic cyst, and each of these women underwent a uterus-preserved cystectomy by laparoscopy. A final diagnosis of endometriosis was confirmed and supported by the subsequent histology. The endometriosis staging was in accordance with the revised American Fertility Society classification (10). Among the 55 women with endometriosis, 12 were classed as fertile with stage I $(n=5)$, stage II $(n=4)$, stage III $(n=2)$ or stage IV $(n=1)$ disease. The other 43 women were infertile with stage I $(n=13)$, stage II $(n=16)$, stage III $(n=9)$ or stage IV $(n=5)$ disease. Information about pelvic pain, dysmenorrhea and average ovarian endometriotic cyst diameter was taken from the patients' clinical records. The control subjects were 47 women (aged 28-38 years), undergoing surgery for tubal ligation (36 cases) or hysterectomy for benign indications (11 cases), who had no visible evidence of endometriosis. The mean age of the women with endometriosis and control women was $31.7 \pm 3.8$ and $30.9 \pm 2.9$ years, respectively, and no difference in age was observed between the two groups $(\mathrm{P}>0.05)$.

Blood samples were obtained for the measurement of the serum $\mathrm{E}_{2}$ and progesterone $(\mathrm{P})$ concentrations on the morning of the day of surgery, and endometrial tissues were simultaneously obtained via Pipelle ${ }^{\circledR}$ endometrial curettage (CCD Laboratories, Paris, France) on days 6-10 (proliferative phase) or 18-26 (secretory phase) of the menstrual cycle. Samples at were collected different phases of the menstrual cycle from different patients in order to avoid the possible effects of the first sample on subsequent samples. Following collection, the endometrial tissues were rapidly either snap-frozen in liquid nitrogen and stored at $-80^{\circ} \mathrm{C}$ for the subsequent extraction of protein and mRNA. According to the Noyes pathological diagnosis (hematoxylin and eosin staining) the endometrial samples were assigned to one of four groups: Proliferative phase of endometriosis $(n=26)$, secretory phase of endometriosis $(n=29)$, proliferative phase of control $(n=24)$ and secretory phase of control $(n=23)$.

Western blot analysis. Cells were lysed in Laemmli lysis buffer (Bio-Rad Laboratories, Inc., Hercules, CA, USA) containing $1 \%$ Triton X-100 and scraped with a cell lifter. Equal quantities of protein $(25 \mu \mathrm{l})$ were separated using $8 \%$ SDS-PAGE and transferred to Immobilon ${ }^{\mathrm{TM}}-\mathrm{P}$ membranes (Millipore, Billerica, MA, USA). Blocking was performed with 5\% skimmed dried milk at $4^{\circ} \mathrm{C}$ overnight, and the membranes were then incubated with primary antibodies (sc-20121; Santa Cruz Biotechnology, Inc., Santa Cruz, CA, USA) at room temperature for $2 \mathrm{~h}$. Following incubation, the membranes were washed three times with $1 \mathrm{X}$ phosphate-buffered saline (PBS) in $0.1 \%$ Tween-20 and incubated with the respective horseradish peroxidase-conjugated secondary antibodies (1:10,000; P0488; Dako Cytomation, Inc., Carpinteria, CA, USA). Signal visualization was conducted through enhanced chemiluminescence (GE Healthcare Life Sciences, Little Chalfont, UK). The bound antibody was detected by using an
ECL detection reagent (Santa Cruz Biotechnology, Inc.). The bands were scanned by using Quantity One software (Bio-Rad Laboratories, Inc.). Normalized densities were determined by using the ratio of the band density of MIF to the band density of -actin.

Reverse transcription-polymerase chain reaction (RT-PCR) analysis. Total RNA was isolated with TRIzol ${ }^{\circledR}$ reagent (Invitrogen Life Technologies, Carlsbad, CA, USA) and then reverse transcribed using oligo(dT) primers and SuperScript ${ }^{\circledR}$ II reverse transcriptase (Invitrogen Life Technologies). The PCR primers used were as follows: MIF forward, 5'-TCAAGTCAG CAACGTGGAAG-3' and reverse, 5'-TATCGAGGCTGTGTC GACTG-3'; GAPDH forward, 5'-AGCCATGTACGTAGCCAT CC-3' and reverse, 5'-CTCTCAGCTGTGGTGGTGAA-3'. PCR was performed in a Bio-Rad DNA Engine Tetrad 2 Peltier thermal cycler (Bio-Rad Laboratories, Inc.) under the following conditions: One cycle of $95^{\circ} \mathrm{C}$ for $5 \mathrm{~min}$; 40 cycles of $95^{\circ} \mathrm{C}$ for $30 \mathrm{sec}, 54^{\circ} \mathrm{C}$ for $30 \mathrm{sec}$ and $72^{\circ} \mathrm{C}$ for $30 \mathrm{sec}$; and finally one cycle of $72^{\circ} \mathrm{C}$ for $10 \mathrm{~min}$. The PCR products $(10 \mu \mathrm{l})$ were subsequently mixed with $2 \mu$ l loading buffer and electrophoretically separated on $2 \%$ agarose gel for visualization with ethidium bromide. GADPH was used an endo-reference control.

Measurements of serum $E_{2}$ and $P$ levels. The concentrations of $E_{2}$ and $P$ in the sera of the patients were measured with a double-antibody radioimmunoassay (Diagnostic Products Corp., Los Angeles, CA, USA).

Primary cell culture. All biopsies were collected in the operating room under sterile conditions. The tissues were rinsed with PBS, and the endometrium was dissected free from the underlying myometrium or parenchyma. The tissue was sliced into $\sim 1-\mathrm{mm}^{3}$ fragments, which were subsequently incubated with collagenase $(2 \mathrm{mg} / \mathrm{ml}$; Sigma-Aldrich, St. Louis, MO, USA) at $37^{\circ} \mathrm{C}$ in $5 \% \mathrm{CO}_{2}$ for $60-90 \mathrm{~min}$. DNase $(2 \mathrm{mg} / \mathrm{ml}$; Sigma-Aldrich) was then added. The endometrial cells were separated from the debris by filtration using narrow-gauge sieves with a $70-\mu \mathrm{m}$ mesh filter, plated and allowed to adhere to plastic dishes for $\sim 2 \mathrm{~h}$. Any blood cells and debris were subsequently removed by rinsing with PBS. All cells were starved for 2 days prior to treatment with $1 \times 10^{-7} \mathrm{M} \mathrm{E}_{2}$. After $24 \mathrm{~h}$, the cells were harvested and used for western blotting and RT-PCR analysis.

Statistical analysis. All data were normally distributed. Statistical analysis of the ratios of MIF to the end reference was performed using one-way analysis of variance with SPSS 11.5 software (SPSS, Inc., Chicago, IL, USA). Results are expressed as the mean \pm standard deviation. Multiple comparisons were performed using the Bonferroni procedure, while single comparisons were conducted using the Student's t-test. $\mathrm{P}<0.05$ was considered to indicate a statistically significant difference. Linear regression was used to the analyze correlation between MIF expression and the serum $\mathrm{E}_{2}$ and $\mathrm{P}$ levels.

\section{Results}

A difference in human endometrial MIF expression can be found between women with and without endometriosis. 

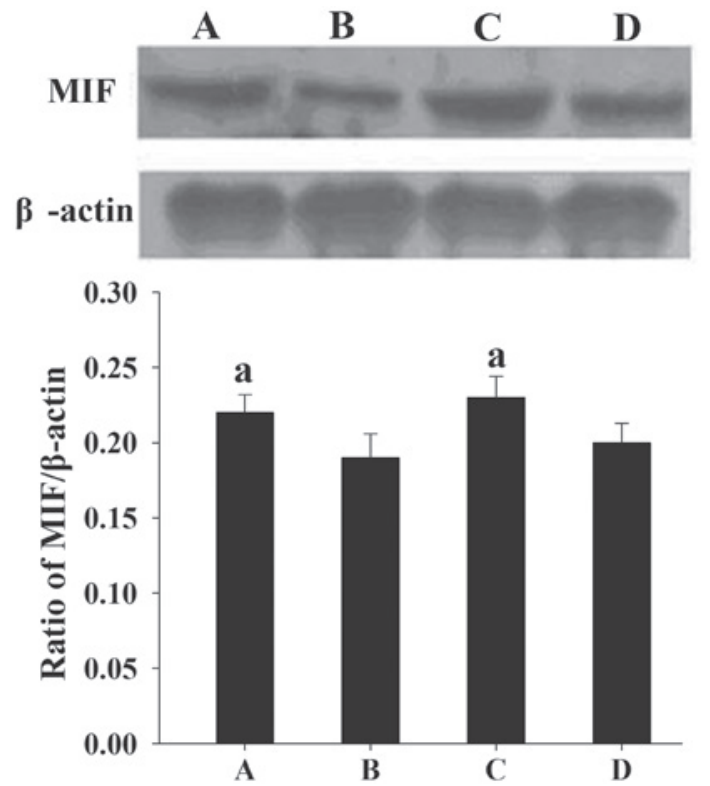

Figure 1. Assessment of MIF protein expression in endometrial tissues by western blotting. (A and B) Proliferative endometrium from (A) women with endometriosis and (B) controls. (C and D) Secretory endometrium from (C) women with endometriosis and (D) controls. The normalized density was analyzed using internal $\beta$-actin as a reference. Results are presented as the mean \pm standard deviation. ${ }^{a} \mathrm{P}<0.05$, compared with the normalized density of MIF protein in the secretory endometrial tissues of the controls. MIF, macrophage migration inhibitory factor.

Following the normalization of each band of MIF from different samples to $\beta$-actin, it was found that the mean levels of MIF protein expression in women with endometriosis at the proliferative and secretory phases $(0.22 \pm 0.012$ and $0.23 \pm 0.014$, respectively) were significantly higher than those in the control women $(0.19 \pm 0.016$ and $0.20 \pm 0.013$, respectively) $(\mathrm{P}<0.05)$ (Fig. 1).

Similar to the results from the western blotting, semi-quantitative PCR analysis showed that the mean levels of MIF mRNA expression in women with endometriosis at the proliferative and secretory phases $(0.21 \pm 0.011$ and $0.21 \pm 0.014$, respectively) were significantly higher than those in the control women $(0.18 \pm 0.009$ and $0.19 \pm 0.008$, respectively) $(\mathrm{P}<0.05)$ (Fig. 2).

Correlation between human endometrial MIF expression and the serum levels of $E_{2}$. The serum $E_{2}$ concentration was measured at the proliferative and secretory phases of the menstrual cycle, and the serum $\mathrm{P}$ concentration was determined at the secretory phase. All patients in the present study showed a normal ovarian steroid hormone profile. The serum $\mathrm{E}_{2}$ concentrations ranged from 102.64 to $785.29 \mathrm{pmol} / \mathrm{l}$, and a positive correlation was found between the serum $\mathrm{E}_{2}$ level and MIF protein expression (Fig. 3). As shown in Fig. 4, a positive correlation was also noted between the serum $\mathrm{E}_{2}$ level and MIF mRNA expression.

Regulation of MIF expression by $E_{2}$ in human endometrial cells. Following treatment with $\mathrm{E}_{2}$, an elevated expression of MIF protein (Fig. 5) and mRNA (Fig. 6) was observed in the cultured endometrial cells compared with the untreated endometrial cells $(\mathrm{P}<0.05)$.

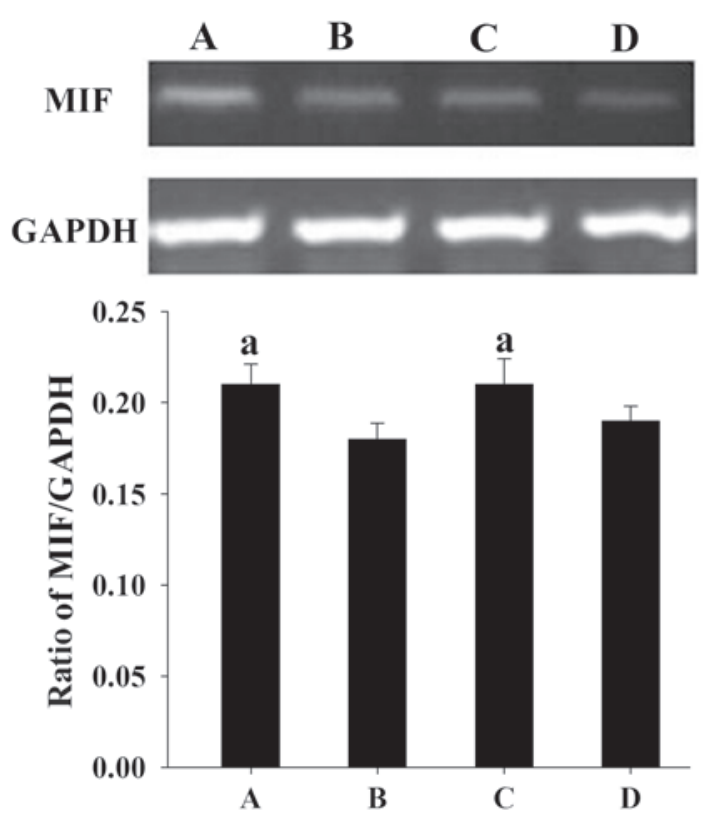

Figure 2. Assessment of MIF mRNA expression by reverse transcription-polymerase chain reaction analysis in endometrial samples. A 399-bp product of MIF mRNA was visualized with ethidium bromide following agarose gel electrophoresis. GAPDH (452 bp) was used as a control to assess the amount of RNA in each sample. (A and B) Proliferative endometrium from (A) women with endometriosis and (B) controls. (C and D) Secretory endometrium from (C) women with endometriosis and (D) controls. Quantification of the MIF mRNA level was performed using internal GAPDH as a reference. Results are presented as the mean \pm standard deviation. ${ }^{a} \mathrm{P}<0.05$, compared with the normalized density of MIF mRNA in the secretory endometrial tissues of the controls. MIF, macrophage migration inhibitory factor.

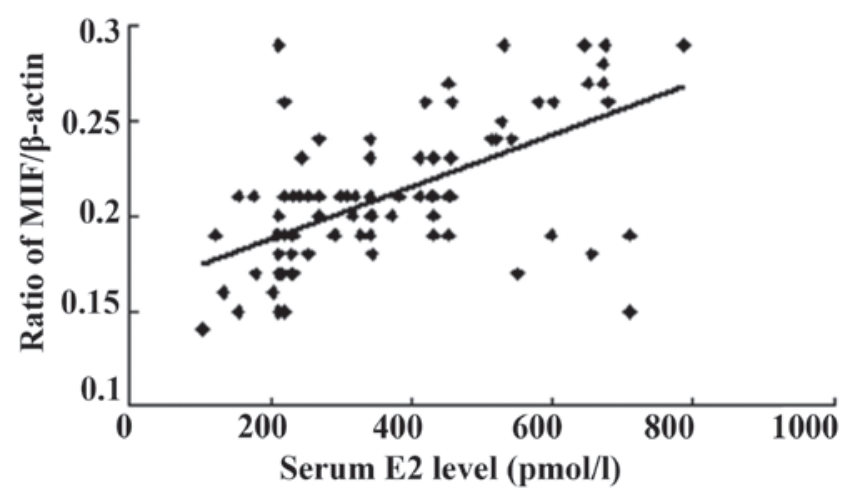

Figure 3. Normalized endometrial MIF protein concentration is correlated with the serum $\mathrm{E}_{2}$ level $(\mathrm{n}=102 ; \mathrm{r}=0.611, \mathrm{P}<0.01)$. MIF, macrophage migration inhibitory factor; $\mathrm{E}_{2}, 17 \beta$-estradiol.

\section{Discussion}

In the present study, MIF protein and mRNA levels were found to be increased in the endometrial tissues from women with endometriosis. In a study by Arcuri et al (11), no significant differences in MIF levels were found across the menstrual cycle. These results are consistent with those of previous studies (11-13).

MIF, which is a $12.5-\mathrm{kDa}$ cytokine that inhibits the migration and chemotaxis of macrophages $(14,15)$, was first identified as a T-cell-derived lymphokine $(16,17)$. MIF exerts a wide range of immunostimulatory and proinflammatory 


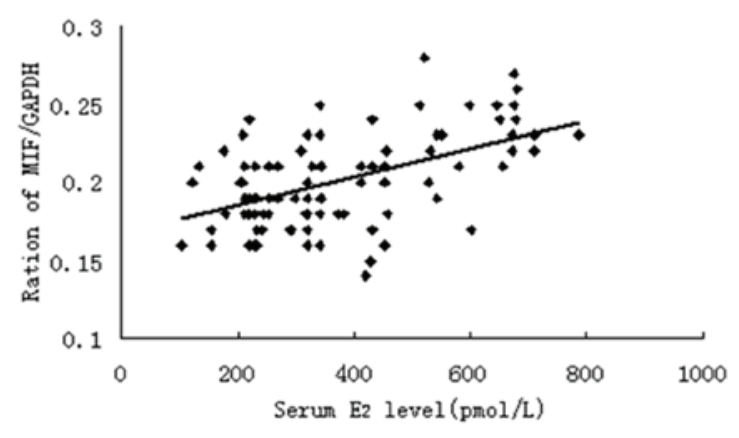

Figure 4. Normalized endometrial MIF mRNA concentration is correlated with the serum $\mathrm{E}_{2}$ level $(\mathrm{n}=102 ; \mathrm{r}=0.522, \mathrm{P}<0.01)$. MIF, macrophage migration inhibitory factor; $\mathrm{E}_{2}, 17 \beta$-estradiol.
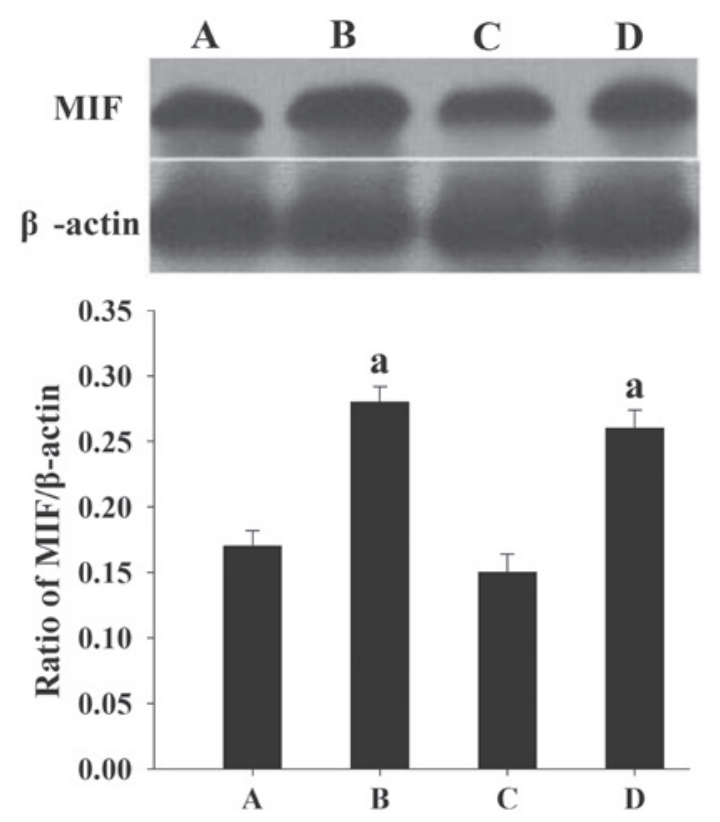

Figure 5. Effect of $\mathrm{E}_{2}$ on MIF protein expression in endometrial cells, as assessed by western blotting. (A) Untreated and (B) $\mathrm{E}_{2}$-treated endometrial cells from women with endometriosis. (C) Untreated and (D) $\mathrm{E}_{2}$-treated endometrial cells from women without endometriosis. The normalized density was analyzed using internal $\beta$-actin as the reference. Results are presented as the mean \pm standard deviation. ${ }^{a} \mathrm{P}<0.05$, compared with the normalized density of MIF proteins in the untreated endometrial tissues of the controls. MIF, macrophage migration inhibitory factor; $\mathrm{E}_{2}, 17 \beta$-estradiol.

effects (18) and is produced not only by activated T cells and macrophages, but also by the endothelial and epithelial cells of several organs (19-21). MIF is important in tumor growth and angiogenesis (22-24), and has been suggested to have a pivotal function in cell proliferation and differentiation, angiogenesis and wound healing $(18,25)$. Endometriosis, although not neoplastic, shares features with malignant cells and the metastasis in endometriosis may include certain mechanisms proposed in cancer (26). The higher expression of MIF in endometriosis could be associated with the fact that the endometrial cells from women with endometriosis have a predisposition to adhere and grow outside of the uterus (4).

In the present study it was found that the expression of MIF in endometrial tissues was correlated with the serum level of $\mathrm{E}_{2}$. This result suggests that estrogen contributes to
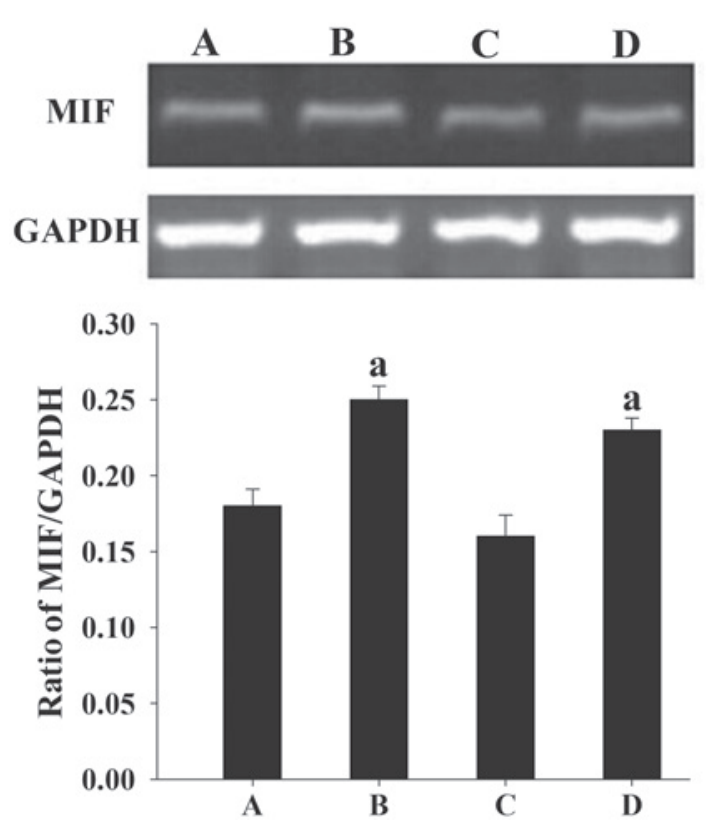

Figure 6. Effect of $\mathrm{E}_{2}$ on MIF mRNA expression in endometrial samples, as assessed by reverse transcription-polymerase chain reaction analysis. A 399-bp product of MIF mRNA was visualized with ethidium bromide following agarose gel electrophoresis. GAPDH (452 bp) was used as a control to assess the amount of RNA in each sample.(A) Untreated and (B) $E_{2}$-treated endometrial cells from women with endometriosis. (C) Untreated and (D) $E_{2}$-treated endometrial cells from women without endometriosis. Quantification of the MIF mRNA level was performed using internal GAPDH as a reference. Results are presented as the mean \pm standard deviation. ${ }^{\mathrm{a}} \mathrm{P}<0.05$, compared with the normalized density of MIF proteins in the untreated endometrial tissues of the controls. MIF, macrophage migration inhibitory factor; $\mathrm{E}_{2}$, $17 \beta$-estradiol.

the regulation of MIF in endometrial tissue; however, there are contradictory theories regarding the association between estrogen and MIF. It has previously been demonstrated that MIF expression is upregulated in the wound healing process of estrogen-deficient mice, and that estrogen treatment can directly inhibit MIF production by murine macrophages (27). Houdeau et al (28) found that estrogen could decrease MIF production in the female rat colon, which may have affected the susceptibility of the colon to inflammation. In addition, Hsieh et al (29) revealed that the protective effects of estrogen on lung injury following traumatic hemorrhage were mediated via the downregulation of lung MIF production. Regarding the endometrium, however, the expression of MIF has been shown to be a dynamic process. At the start of the proliferative phase of the menstrual cycle, the MIF protein levels are low. MIF expression then increases during the mid-late proliferative phase and reaches a maximum level around ovulation, prior to undergoing a progressive reduction to moderate levels in the mid-secretory phase and a final further reduction in the late secretory phase (30). These changes are synchronous with the fluctuation of estrogen and are consistent with the observations in the present study. Any inconsistencies in data concerning estrogen and MIF levels are likely due to inter-tissue/-cellular differences.

A previous study demonstrated that estrogen is able to act through nonclassical membrane receptors, leading to rapid intracellular responses (31). The exact pathway underlying the regulation of MIF by estrogen requires further study. 
In the present study it was found that MIF expression in the endometrial cells from women with endometriosis was more sensitive to $\mathrm{E}_{2}$ than the MIF expression in endometrial cells from women without endometriosis. During the menstrual cycle, the human endometrium undergoes profound and dynamic changes that are accordant with the changes in the levels of steroid hormones, such as $E_{2}$, which is a critical hormone that favors the development and maintenance of endometriosis (9). The oversensitive upregulation of MIF expression may contribute to the pathogenesis and progression of endometriosis.

In conclusion, the present study has shown for the first time, to the best of our knowledge, that MIF expression in the endometrial tissues of women with endometriosis is significantly increased, that MIF expression is associated with the level of $E_{2}$, and that MIF expression in endometrial cells from women with endometriosis is more sensitive to $E_{2}$ than that in cells from women without endometriosis. These findings suggest that MIF contributes to the pathogenesis of endometriosis and is regulated by steroid hormones. The mechanisms by which increased endometrial MIF levels in endometriosis affect the reproductive function of the patients require further elucidation. In addition, further investigations into the use of MIF as a clinical diagnostic marker and as a therapeutic target are warranted.

\section{Acknowledgements}

This study was supported by a grant from the National Natural Science Foundation of China (no. 81100407).

\section{References}

1. Sampson JA: Peritoneal endometriosis due to the menstrual dissemination of endometrial tissue into the peritoneal cavity. Am J Obstet Gynecol 14: 422-469, 1927.

2. Strathy JH, Molgaard CA, Coulam CB and Melton JL III: Endometriosis and infertility: A laparoscopic study of endometriosis among fertile and infertile women. Fertil Steril 38 667-672, 1982.

3. Kao L, Germeyer A, Tulac S, et al: Expression profiling of endometrium from women with endometriosis reveals candidate genes for disease-based implantation failure and infertility. Endocrinology 144: 2870-2881, 2003.

4. Ulukus M, Cakmak H and Arici A: The role of endometrium in endometriosis. J Soc Gynecol Investig 13: 467-476, 2006.

5. Larson DF and Horak K: Macrophage migration inhibitory factor: Controller of systemic inflammation. Crit Care 10: 138, 2006.

6. Bernhagen J, Calandra T, Mitchell RA, et al: MIF is a pituitary-derived cytokine that potentiates lethal endotoxaemia. Nature 365: 756-759, 1993.

7. Berkkanoglu M and Arici A: Immunology and endometriosis. Am J Reprod Immunol 50: 48-59, 2003.

8. Akoum A, Metz CN, Al-Akoum M and Kats R: Macrophage migration inhibitory factor expression in the intrauterine endometrium of women with endometriosis varies with disease stage, infertility status, and pelvic pain. Fertil Steril 85: 1379-1385, 2006.

9. Deroo BJ and Korach KS: Estrogen receptors and human disease. J Clin Invest 116: 561-570, 2006.

10. Brosens IA, Cornillie F, Koninckx P and Vásquez G: Evolution of the Revised American Fertility Society Classification of Endometriosis. Fertil Steril 44: 714-716, 1985.
11. Arcuri F, Ricci C, Ietta F, et al: Macrophage migration inhibitory factor in the human endometrium: Expression and localization during the menstrual cycle and early pregnancy. Biol Reprod 64: 1200-1205, 2001

12. Lin W, Chen S, Li M, Wang B, Qu X and Zhang Y: Expression of macrophage migration inhibitory factor in human endometriosis: relation to disease stage, menstrual cycle and infertility. J Obstet Gynaecol Res 36: 344-351, 2010.

13. Akoum A, Metz CN, Al-Akoum M and Kats R: Macrophage migration inhibitory factor expression in the intrauterine endometrium of women with endometriosis varies with disease stage, infertility status, and pelvic pain. Fertil Steril 85: 1379-1385, 2006.

14. Calandra $\mathrm{T}$ and Roger $\mathrm{T}$ : Macrophage migration inhibitory factor: A regulator of innate immunity. Nat Rev Immunol 3: 791-800, 2003.

15. Hermanowski-Vosatka A, Mundt SS, Ayala JM, et al: Enzymatically inactive macrophage migration inhibitory factor inhibits monocyte chemotaxis and random migration. Biochemistry 38: 12841-12849, 1999.

16. David JR: Delayed hypersensitivity in vitro: Its mediation by cell-free substances formed by lymphoid cell-antigen interaction. Proc Natl Acad Sci USA 56: 72-77, 1966.

17. Bloom BR and Bennett B: Mechanism of a reaction in vitro associated with delayed-type hypersensitivity. Science 153: 80-82, 1966.

18. Nishihira J: Macrophage migration inhibitory factor (MIF): Its essential role in the immune system and cell growth. J Interferon Cytokine Res 20: 751-762, 2000.

19. Donnelly SC, Haslett C, Reid PT, et al: Regulatory role for macrophage migration inhibitory factor in acute respiratory distress syndrome. Nature Med 3: 320-323, 1997.

20. Imamura K, Nishihira J, Suzuki M, et al: Identification and immunohistochemical localization of macrophage migration inhibitory factor in human kidney. IUBMB Life 40: 1233-1242, 1996.

21. Suzuki M, Sugimoto H, Nakagawa A, Tanaka I, Nishihira J and Sakai M: Crystal structure of the macrophage migration inhibitory factor from rat liver. Nat Struct Biol 3: 259-266, 1996.

22. Chesney J, Metz C, Bacher M, Peng T, Meinhardt A and Bucala R: An essential role for macrophage migration inhibitory factor (MIF) in angiogenesis and the growth of a murine lymphoma. Mol Med 5: 181-191, 1999.

23. Bingle L, Brown NJ and Lewis CE: The role of tumour-associated macrophages in tumour progression: Implications for new anticancer therapies. J Pathol 196: 254-265, 2002.

24. Ogawa H, Nishihira J, Sato Y, et al: An antibody for macrophage migration inhibitory factor suppresses tumour growth and inhibits tumour-associated angiogenesis. Cytokine 12: 309-314, 2000.

25. Nishihira J, Ishibashi T, Fukushima T, Sun B, Sato Y and Todo S: Macrophage migration inhibitory factor (MIF): Its potential role in tumor growth and tumor-associated angiogenesis. Ann NY Acad Sci 995: 171-182, 2003.

26. Zeitvogel A, Baumann R and Starzinski-Powitz A: Identification of an invasive, $\mathrm{N}$-cadherin-expressing epithelial cell type in endometriosis using a new cell culture model. Am J Pathol 159: 1839-1852, 2001

27. Ashcroft GS, Mills SJ, Lei K, et al: Estrogen modulates cutaneous wound healing by downregulating macrophage migration inhibitory factor. J Clin Invest 111: 1309-1318, 2003.

28. Houdeau E, Moriez R, Leveque M, et al: Sex steroid regulation of macrophage migration inhibitory factor in normal and inflamed colon in the female rat. Gastroenterology 132: 982-993, 2007.

29. Hsieh YC, Frink M, Hsieh CH, et al: Downregulation of migration inhibitory factor is critical for estrogen-mediated attenuation of lung tissue damage following trauma-hemorrhage. Am J Physiol Lung Cell Mol Physiol 292: L1227-L1232, 2007.

30. Kats R, Al-Akoum M, Guay S, Metz C and Akoum A: Cycle-dependent expression of macrophage migration inhibitory factor in the human endometrium. Hum Reprod 20: 3518-3525, 2005.

31. Ropero AB, Soria B and Nadal A: A nonclassical estrogen membrane receptor triggers rapid differential actions in the endocrine pancreas. Mol Endocrinol 16: 497-505, 2002. 